\title{
Disposable graft splint in penetrating keratoplasty
}

\author{
P. HARDY SMITH \\ Melbourne
}

Jones and Rice (1968) have demonstrated experimentally that the endothelium of the donor corneal disc may be subjected to considerable mechanical trauma during penetrating keratoplasty by the more commonly used techniques. From these experimental observations they have developed a method in which the corneal disc is sutured over a cushion of air, thus obviating much of the endothelial trauma inseparable from other techniques of penetrating keratoplasty. Their paper referred to keratoplasty in aphakic bullous keratopathy, but the protection of the endothelium is of equal importance in all types of penetrating keratoplasty in order to obtain a clear graft.

One of the difficulties of using the technique is that of maintaining the bubble of air in the anterior chamber while direct sutures are being inserted into the host-graft junction. The description of the operation by Jones and Rice calls for the use of two overlay sutures to hold the graft in place while the direct sutures are being placed.

It is often found that if such overlay sutures are tightened sufficiently to provide a reasonable close host-donor contact, there is a considerable tendency for the host cornea to buckle with consequent difficulty in maintaining an air seal whilst inserting the continuous ro/o nylon suture. If the overlay sutures are only tightened enough to produce host-donor contact without buckling the host cornea, then such contact frequently does not seem to be sufficient to prevent loss of air whilst the continuous suture is being inserted. It is felt that the repeated loss of air from the anterior chamber, with recurrent contact of the lens or vitreous face with the donor endothelium, is likely to produce the very complication that the technique was designed to avoid, namely damage to the donor endothelium.

Various modifications of the technique were designed to avoid this situation and provide a more air-tight seal while the continuous suture was being inserted, but the use of the splint to be described has been found to give uniformly satisfactory results.

\section{Construction of splint}

Circular rings are made of $27 \mathrm{~g}$. steel wire of diameters 12,13 , and $14 \mathrm{~mm}$. similar to Flieringa rings but of smaller diameter. Each ring is placed on a contact lens mould of $8 \mathrm{~mm}$. radius, and six strands of $7 / 0$ black silk are then stretched across the dome of the mould and bonded at each end with waterproof adhesive to the steel ring equidistant from the other strands: the strands cross at a common point at the apex of the contact lens mould and are bonded together at their point of crossing. The final effect resembles a cartwheel, the rim consisting of the steel ring and the spokes consisting of the strands of $7 / 0$ suture material (Fig. I). When taken from the mould the spokes are naturally quite lax. 


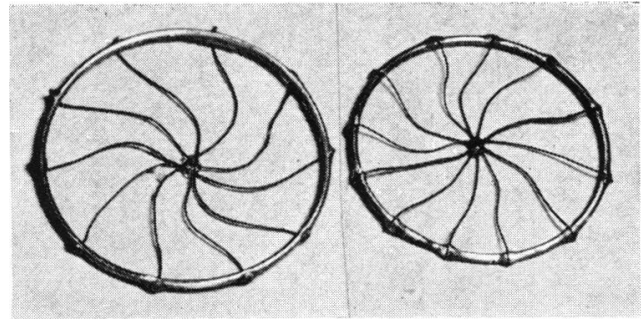

FIG. I Graft splints

\section{Operative technique}

The host eye is prepared as described by Jones and Rice (1969). If the eye to be grafted is aphakic, then preoperative mannitol must be used to produce reduction of vitreous volume and a Flieringa ring must be sutured just anterior to the insertion of the rectus muscles. Anterior chamber paracentesis is performed to enable insertion of sterile air and if the eye is aphakic, to study the reaction of the vitreous face on collapsing the anterior chamber. The size of the corneal disc to be removed is then outlined with the trephine. The diameter of the cornea is measured and a splint selected which is $\mathrm{I}$ or $2 \mathrm{~mm}$. larger in diameter than the cornea. Four $7 / 0$ sutures are inserted in the sclera at 3,6, 9, and 12 o'clock posterior to the limbus and left long, and the splint is placed on the eye so that the ring overlies the site of each suture (Fig. 2). The lateral suture is then tied to secure the ring to the sclera and the splint can then be turned over, using this suture as a fixed point so that it lies clear of the cornea on the lateral side of the eye (Fig. 3).

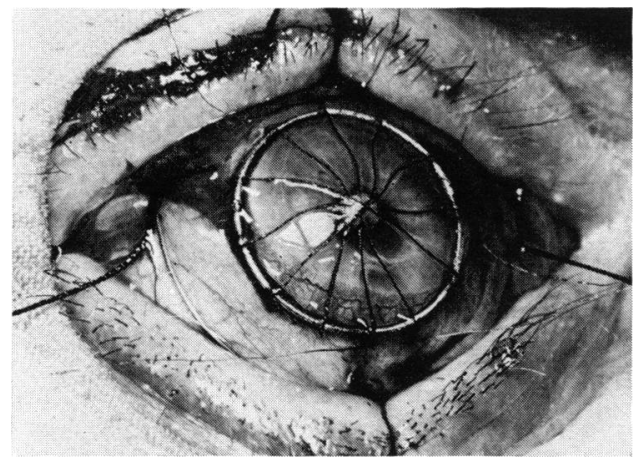

FIG. 2 Graft splint placed on cornea and lateral retaining suture tied. The Flieringa ring can also be seen

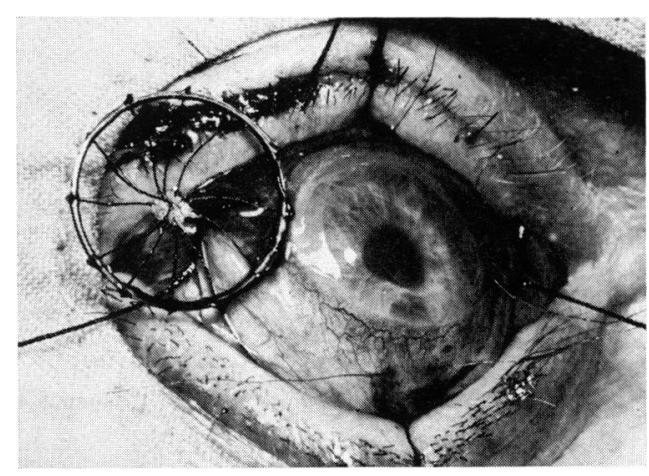

FIG. 3 Graft splint rotated clear of cornea preparatory to trephination

The donor disc is then trephined over a cushion of air in the usual way and left lying on this air bubble until required. Turning again to the host cornea, the anterior chamber is collapsed by removing aqueous through the paracentesis site, and is then re-formed with sterile air. The cornea can now be trephined and the disc removed (Fig. 4, overleaf).

The donor disc is placed in the host eye and the splint is rotated back to overlie the cornea, its rim being secured by the other three sutures which have been previously placed in the sclera.

The anterior chamber is deepened with air injected through the paracentesis site, and because the spokes of the splint have an effective radius of curvature of $8 \mathrm{~mm}$. when taut it will be found that the normal curvature of the cornea is restored without deformation of the host-donor junction (Fig. 5 , overleaf).

Four interrupted Io/o sutures are placed at 3,6, 9, and I 2 o'clock to prevent rotation of the graft while the continuous suture is being inserted. 


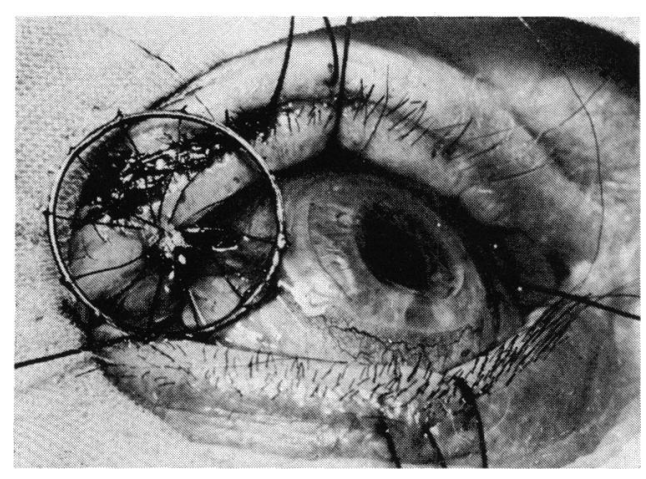

FIG. 4 Corneal disc removed from aphakic eye

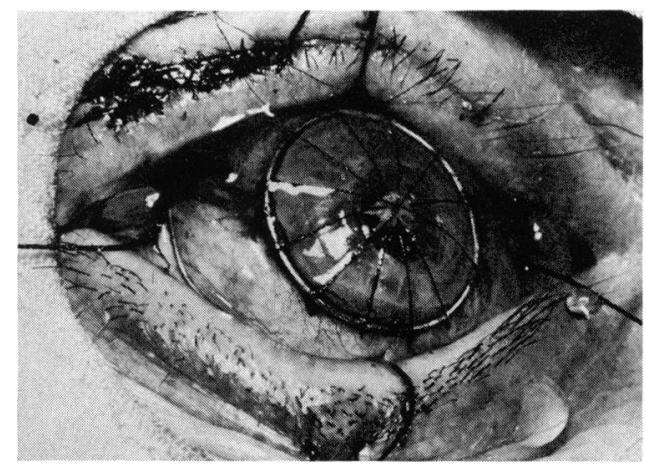

FIG. 5 Graft splint rotated back to overlie donor disc and remaining retaining sutures tied. Anterior chamber re-formed with air

Continuous suturing is now begun at 6 o'clock, using a double armed ro/o nylon suture and carrying the suture round the nasal half with one arm and the temporal half with the other so that the two ends can be tied at 12 o'clock. As the spokes of the splint cross the host-donor junction at twelve points it will be found that, with careful suturing, loss of air and collapse of the anterior chamber do not occur. The air can then be withdrawn through the paracentesis site and the anterior chamber re-formed with balanced salt solution.

The splint is then removed by cutting the spokes and the retaining sutures with a razor blade fragment.

\section{Discussion}

The use of a disposable graft splint as described above, with a rigid outer rim and flexible spokes with an effective radius of curvature of $8 \mathrm{~mm}$., obviates much of the difficulty associated with the technique of penetrating keratoplasty using an air cushion to protect the donor endothelium, in particular the problem of preventing loss of air from the anterior chamber while the direct host-donor sutures are being inserted. The splint can be constructed in various diameters, with various radii of curvature of the spokes, and any number of spokes to allow for different situations. In general it will be found that a radius of curvature of the spokes of $8 \mathrm{~mm}$. is adequate for most cases, as the spokes are flexible and can be well adapted to radii of corneal curvature of slightly more or slightly less than $8 \mathrm{~mm}$. The spokes must be made of material which can be cut easily as they must be severed to remove them from beneath the continuous suture at the conclusion of the operation: it has been found that $7 / 0$ silk is quite satisfactory for this purpose.

\section{Summary}

A disposable splint is described for use in the "air cushion" technique of penetrating keratoplasty.

I am grateful to Optical Prescriptions Spectacle Makers Pty. Ltd. for construction of the rings which form the rim of the splint.

\section{References}

JONES, B. R., and RICE, N. s. c. (I968) Trans. ophthal. Soc. U.K., 88, 343

(1969) In "Corneo-Plastic Surgery: Proc. II int. Corneo-plastic Conference, London 1967",

ed. P. V. Rycroft, p. 307, 3 I I. Pergamon Press, Oxford 